\title{
Sistemas de gestión de calidad en el área de enfermería; un antes y un después en el mundo de las residencias para personas mayores
}

\author{
Pedro Andreo ${ }^{1}$, Carmelo Gómez ${ }^{1,2}$, Elena Carrasco ${ }^{1}$, Inmaculada \\ Martínez-Escámez ${ }^{1}$, Inmaculada Alguazas ${ }^{1}$ y Francisca Andreo ${ }^{1}$ \\ ${ }^{1}$ Asociación Edad Dorada Mensajeros de la Paz Murcia (España); \\ ${ }^{2}$ Universidad Católica San Antonio de Murcia (España)
}

\begin{abstract}
Desarrollar e intentar alcanzar una elevada calidad en el servicio prestado por los profesionales de enfermería de los centros geriátricos mediante la implantación de sistemas de gestión de calidad es tema de actualidad, hecho este probado ya en otros trabajos. Es por esta razón que el objetivo general de este estudio se haya centrado en analizar los efectos más relevantes que surten de la instauración de una forma de gestionar la calidad basada en la Norma UNE EN ISO 9001:2008 en el área de enfermería de cuatro residencias de ancianos. Se ha llevado a cabo un análisis de más de 200 encuestas de satisfacción respecto del servicio de enfermería de cuatro residencias de personas mayores, así como una revisión bibliográfica de los resultados obtenidos tras la implantación de un sistema de gestión de calidad en el área de enfermería de residencias geriátricas. Apreciamos resultados muy positivos en el desarrollo diario de las funciones de enfermería de las residencias geriátricas derivados de la utilización de un sistema de gestión de calidad, pues ha mejorado considerablemente la calidad en la práctica clínica. El estudio muestra que la implantación de un sistema de gestión de calidad proporciona una herramienta útil al servicio de enfermería en residencias de personas mayores evidenciándose los efectos positivos que resultan de dicha implantación mediante encuestas de satisfacción e indicadores sanitarios.

Palabras clave: Calidad asistencial, residencia, enfermería, satisfacción.
\end{abstract}

Quality Assurance Systems in Nursing. A turning point in the world of nursing homes. Developing and trying to achieve a high quality in the service nursing personnel provide at nursing homes by means of implementing quality assurance systems is a topic of general interest nowadays. These systems have already proved to be effective in other fields. That is why the focus of this study is to analyse the most relevant effects due to the implementation of a quality assurance method based on standard UNE EN ISO 9001:2008 in the nursing service of four nursing homes. An analysis of more than 200 answers of a satisfaction survey about the nursing service at four nursing homes has been carried out. A study on the available bibliography about the results obtained after having implemented a quality assurance system in the nursing service at nursing homes has also been carried out. Very positive results can be appreciated in the daily nursing practice at nursing homes as a consequence of using a quality assurance system. Clinical practice has improved considerably as a consequence. The study shows quality assurance systems to be a useful tool at the service of nursing practice at nursing homes. Positive effects resulting from the implementation of such quality assurance systems are evident as it can be seen from satisfaction surveys and health indicators.

Keywords: Healthcare quality, nursing home, nursing, satisfaction.

Correspondencia: Pedro Andreo Muñoz. Asociación Edad Dorada Mensajeros de la Paz Murcia. C/ Santiago, 10. C.P.: 30850. Totana, Murcia (España). E-mail: peri-85@ hotmail.com 
Durante las últimas décadas hemos visto como los sistemas de gestión de calidad se han insertado en la cultura de las organizaciones. Este movimiento que se originó principalmente en las empresas industriales está siendo exportado a las áreas de servicios a medida que se difunden sus prácticas y beneficios asociados. Dentro de la geriatría, es el campo de las residencias para personas mayores donde más relevancia está adquiriendo.

Muy conocido por todos es que los cuidados enfermeros han constituido desde sus inicios la piedra base de cualquier institución geriátrica, cosa esta debida a la disposición, competencia y buen saber a la hora de atender a este sector de la población en actual y progresivo aumento. El hecho de dirigir los servicios de una residencia geriátrica desde el punto de vista de los cuidados enfermeros simboliza una pieza fundamental para conseguir el objetivo enfermero de la referida institución. De aquí la importancia de que el personal de enfermería pueda valerse de esta herramienta basada en indicadores sanitarios de calidad para el desarrollo óptimo de sus funciones mejorando la calidad del servicio. La utilización de indicadores como método de trabajo no es una simple tendencia, sino que por el contrario, debe ser entendida como una herramienta que ayude a mejorar el sistema de gestión con el cual se está trabajando. Necesario resulta también destacar que su efecto será mayor cuanto mayor sea el compromiso de los profesionales que prestan el servicio, fusionando un rendimiento ordenado con la audacia de una buena gestión. No menos importancia tiene la formación con el objetivo de capacitar al personal para la correcta observación, estudio y selección de los mencionados indicadores (Llecha, 2008).

La incorporación del sistema de gestión en el cuidado de enfermería implica una serie de acciones que apoyen el desarrollo de los objetivos de la organización en materia sanitaria, favoreciendo la prestación de los cuidados necesarios con un carácter de integralidad, oportunidad y constancia, en un ambiente seguro, eficiente y humano, satisfaciendo las necesidades del residente a fin de mantener o mejorar la calidad de vida del mismo. Probablemente los dos sistemas de gestión de calidad más pretendidos por el sector de las residencias privadas lo forman el modelo EFQM (European Foundation for Quality Management) y las Normas Internacionales de Estandarización (ISO). Ambos modelos han sido insertados a nivel sanitario especialmente hacia la última década (Sociedad Española de Nefrología, 2006), aunque con efectos cada vez más notables y trascendentales. La envergadura que ha significado la incorporación de estos sistemas de gestión de calidad en el marco residencial es indiscutible. Inmensa resulta ser la variedad bibliográfica que hace alusión a la definición del concepto calidad. Destacamos la que le da la Real Academia de la Lengua Española que la define como "Propiedad o conjunto de ellas pertenecientes a una cosa que posibilitan apreciarla como igual, mejor o peor que el resto de su especie". Por otro lado encontramos la definición que le otorga la Norma ISO (Aenor, 2008), "Grado en el cual un grupo de propiedades cumple con los 
requisitos, normalmente implícitos u obligatorios". Para conseguir un correcto aprovechamiento de esta norma en el ámbito sanitario se debe tener en cuenta que la salud no es un concepto medible, por lo que se hace necesario que sea utilizada con pautas de flexibilidad y tolerancia (Cabarcas, 2010).

La aplicación, puesta en marcha y traslado de todo este proceso al ámbito de la enfermería geriátrica puede ser vista como una etapa ardua y engorrosa, pero que con una implantación adecuada de los procedimientos, un interés apropiado por parte de los profesionales de llevarlos a cabo según han sido consensuados y con el objeto primordial de mejorar continuamente el proceso de atención, a medio plazo deslumbrarán los resultados positivos de esta valiosa herramienta traducida en un aumento de la satisfacción de nuestros residentes y familiares, siendo este el objetivo final de nuestras acciones (Manzano, Vázquez, Gutiérrez y Rivero, 2011).

Todos los artículos y estudios revisados coinciden en que la calidad es el desenlace final del proceso enfermero, y enmarcan el ideal de que para conseguirla exitosamente resulta imprescindible implantar un sistema de gestión de calidad que persiga una armonía entre los objetivos institucionales, recursos materiales, demandas del residente y satisfacción tanto de quien provee el cuidado como el de quien lo recibe, así como también coinciden en que al hablar de calidad no hacemos referencia a una simple tendencia actual sino a una forma de trabajar que debe ser incluida en la estructura de su misión.

El presente estudio tiene como objetivo principal analizar los efectos más relevantes que derivan de la implantación de un sistema de gestión de calidad (Norma UNE EN ISO 9001:2008) en el área de enfermería de 4 residencias para personas mayores de la Región de Murcia.

Objetivos específicos:

Conocer la precepción de calidad de residentes y familiares en relación a la capacidad de respuesta del equipo de enfermería.

Conocer la precepción de calidad de residentes y familiares en relación a la preparación y formación del equipo de enfermería.

Conocer la precepción de calidad de residentes y familiares en relación al orden, higiene y confort del servicio de enfermería.

Conocer la precepción de calidad de residentes y familiares en relación a la atención y servicio en general del equipo de enfermería.

\section{MÉTODO}

\section{Participantes}

Se ha tomado como muestra a 340 residentes de cuatro centros geriátricos de la Región de Murcia utilizando como criterio de inclusión presentar una puntuación >24 
en la escala MMSE Mini-mental state examination, (Folstein, Mc Hugh y Fanjiang, 1975), así como a sus respectivos familiares elegidos estos últimos mediante muestreo aleatorio simple.

Las características de los residentes se muestran en la tabla 1 (ver tabla 1) y las características de los residentes con MMSE> 24 se muestran en la tabla 2

Tabla 1. Características de los residentes que conforman la muestra

\begin{tabular}{cll}
\hline & Hombres & Mujeres \\
\hline Residentes, $n(\%)$ & $105(30.9 \%)$ & $235(69.1 \%)$ \\
\hline Edad, media $(D E)$ & $78.38(9.64)$ & $83.38(6.65)$ \\
\hline DE: Desviación Estándar.
\end{tabular}

Tabla 2. Características de los residentes con MMSE> 24

\begin{tabular}{lll}
\hline & Hombres & Mujeres \\
\hline Residentes con MMSE>24, $n(\%)$ & $37(35.2 \%)$ & $73(31.1 \%)$ \\
\hline Edad, media $(D E)$ & $76.57(6.84)$ & $81.8(7.06)$ \\
\hline DE: Desviación Estándar. & &
\end{tabular}

\section{Instrumento}

Como instrumento de recogida de datos utilizamos dos encuestas elaboradas por el equipo de investigadores del estudio. Estas encuestas han sido pasadas desde el año 2010 hasta la actualidad de forma anual, siendo los años 2010 y 2013 los comparados. Para ordenar los datos y posteriormente procesarlos se utilizó Excel, del paquete de Microsoft.

Procedimiento y análisis de datos

Tipo de estudio: Estudio analítico transversal comparativo

Las encuestas consisten en la valoración de 10 ítems a valorar entre una puntuación de 1 a 10 donde:

1 a 2 : deficiente

3 a 4 : mejorable

5 a 6: bueno

7 a 8 : muy bueno

9 a 10: excelente

Los 10 ítems de los que se compone la encuesta se agrupan en 4 bloques: Capacidad de respuesta del equipo de enfermería; Preparación y formación del equipo de enfermería; Orden, higiene y confort del servicio de enfermería; y, Atención y servicio en general del equipo de enfermería.

Para el análisis estadístico se ha utilizado el programa Excel 2010. Para el cálculo de la prevalencia, se ha contabilizado el número total de personas institucionalizadas en los centros en el momento de los cortes. Los resultados del estudio han sido expresados en números absolutos, porcentajes y medias (desviación estándar). 
La representación gráfica de los resultados se ha llevado a cabo mediante tablas de contingencia y diagramas de líneas.

\section{RESULTADOS}

Las características de los residentes se muestran en la tabla 1 y las características de los residentes con MMSE>24 se muestran en la tabla 2.

Tabla 1. Características de los residentes que conforman la muestra

\begin{tabular}{lll}
\hline & Hombres & Mujeres \\
\hline Residentes, $n(\%)$ & $105(30.9 \%)$ & $235(69.1 \%)$ \\
\hline Edad, media (DE) & $78.38(9.64)$ & $83.38(6.65)$ \\
\hline DE: Desviación Estándar
\end{tabular}

Tabla 2. Características de los residentes con MMSE>24

\begin{tabular}{lll}
\hline & Hombres & Mujeres \\
\hline Residentes con MMSE>24, n (\%) & $37(35.2 \%)$ & $73(31.1 \%)$ \\
\hline Edad, media (DE) & $76.57(6.84)$ & $81.8(7.06)$ \\
\hline DE: Desviación Estándar & &
\end{tabular}

De los 110 residentes encuestados en 2010, ninguno percibió la capacidad de respuesta del equipo de enfermería como deficiente, el $8.1 \%$ de ellos opinaron mejorable, el $35.4 \%$ opinaron bueno, y el $36.3 \%$ y $20 \%$ opinaron muy bueno y excelente respectivamente (ver gráfica 1 ).

Gráfica 1. Capacidad de respuesta del equipo de enfermería percibida por los residentes

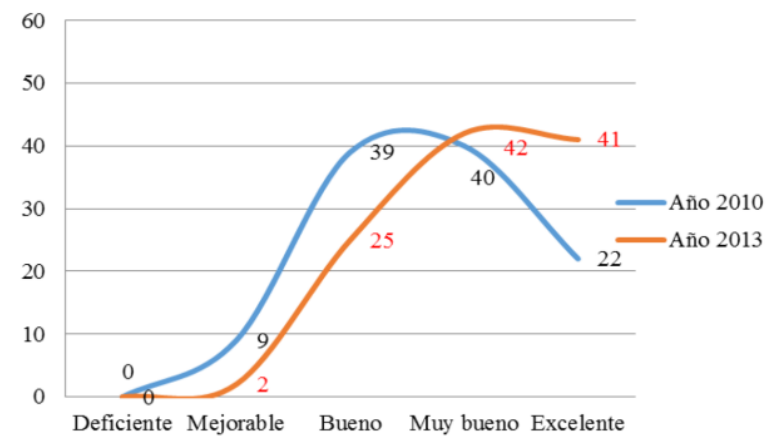

En el año 2013 tampoco ningún residente percibió la capacidad de respuesta del equipo de enfermería como deficiente, mientras que 2 residentes opinaron mejorable, lo que supone el $1.8 \%$; 25 opinaron bueno, lo que supone el $22.7 \%$ y 42 y 41 residentes respectivamente opinaron muy bueno y excelente, lo que supone el $38.1 \%$ y el $37.2 \%$ del total de residentes (ver gráfica 2). 
Gráfica 2. Preparación y formación del equipo de enfermería percibida por los residentes

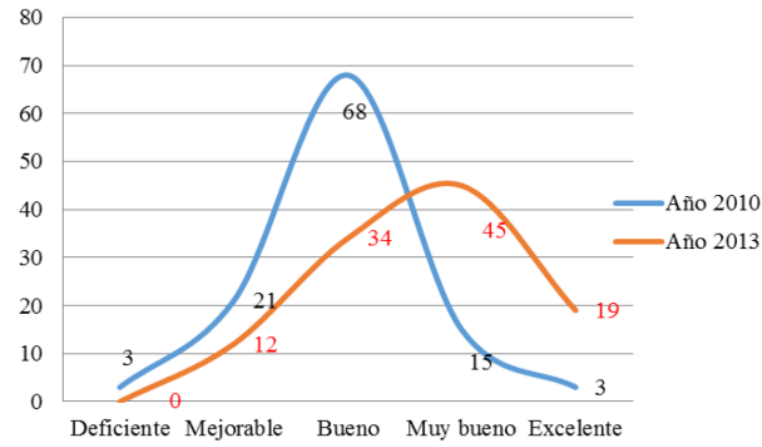

De los 110 residentes encuestados en 2010, 3 percibieron la preparación y formación del equipo de enfermería como deficiente $(2.7 \%)$, el $19.1 \%$ de ellos opinaron mejorable, el $61.8 \%$ opinaron bueno, y el $13.6 \%$ y $2.7 \%$ opinaron muy bueno y excelente respectivamente.

En el año 2013 ningún residente percibió la preparación y formación del equipo de enfermería como deficiente mientras que 12 opinaron mejorable, lo que supone el 10.9\%; 34 opinaron que bueno, lo que supone el $30.9 \%$ y 45 y 19 residentes opinaron muy bueno y excelente respectivamente, lo que supone el $40.9 \%$ y el $17.2 \%$ del total de residentes.

Gráfica 3. Orden, higiene y confort del servicio de enfermería percibida por los residentes

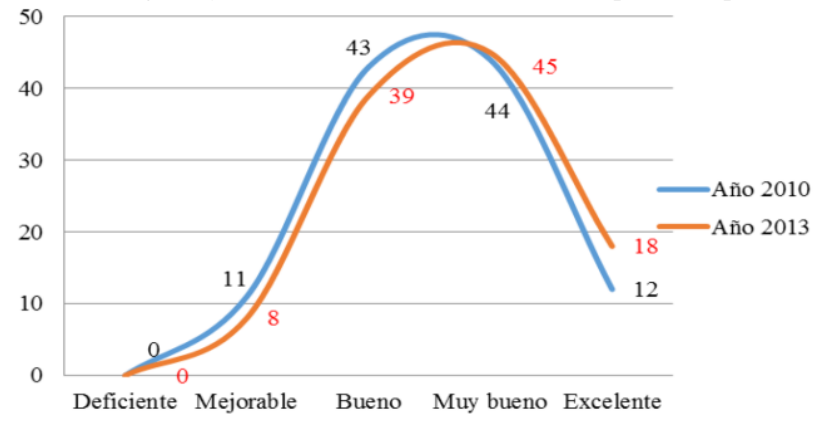

De los 110 residentes encuestados en 2010 ninguno percibió el orden, higiene y confort del servicio de enfermería como deficiente, el $10 \%$ de ellos opinaron mejorable, el $39.1 \%$ opinaron bueno, y el $40 \%$ y $10.9 \%$ opinaron muy bueno y excelente respectivamente.

En el año 2013 ningún residente percibió el orden, higiene y confort del servicio de enfermería como deficiente mientras que 8 opinaron mejorable, lo que 
supone el $7.2 \%$; 39 opinaron que bueno, lo que supone el $35.4 \%$ y 45 y 18 residentes opinaron muy bueno y excelente respectivamente, lo que supone el $40.9 \%$ y el $16.3 \%$ del total de residentes (ver gráfica 3).

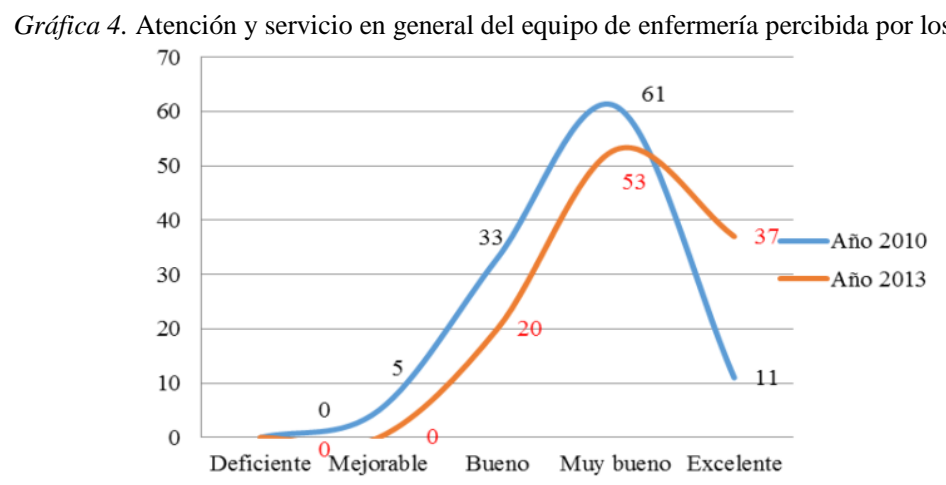

De los 110 residentes encuestados en 2010 ninguno percibió la atención y servicio general del equipo de enfermería como deficiente, el $4.5 \%$ de ellos opinaron mejorable, el $30 \%$ opinaron bueno, y el $55.4 \%$ y $10 \%$ opinaron muy bueno y excelente respectivamente.

En el año 2013 ningún residente percibió la atención y servicio del equipo de enfermería como deficiente ni mejorable; 20 opinaron que bueno, lo que supone el $18.3 \%$. 53 y 37 residentes opinaron muy bueno y excelente respectivamente, lo que supone el $48.1 \%$ y el $33.6 \%$ del total de residentes (ver gráfica 4).

Gráfica 5. Capacidad de respuesta del equipo de enfermería percibida por los familiares

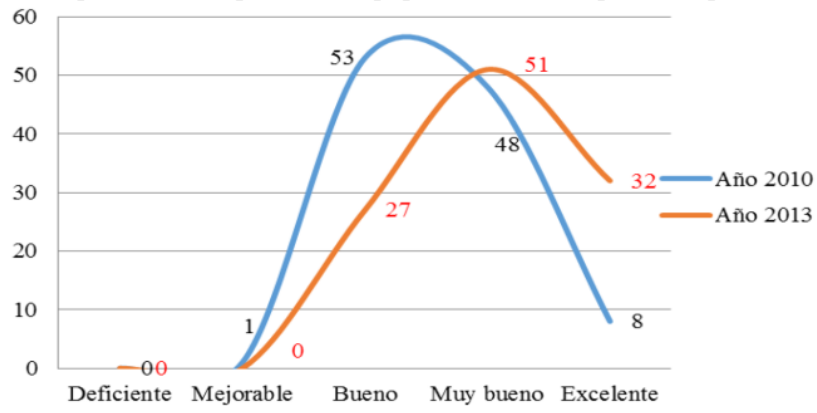

De los 110 familiares encuestados en 2010 ninguno percibió la capacidad de respuesta del equipo de enfermería como deficiente, el $0.9 \%$ de ellos opinaron mejorable, el $48.2 \%$ opinaron bueno, y el $43.6 \%$ y $7.3 \%$ opinaron muy bueno y excelente respectivamente. En el año 2013 ningún familiar percibió la atención y 
servicio del equipo de enfermería como deficiente ni mejorable; 27 opinaron que bueno, lo que supone el 24.5\%. 51 y 32 familiares opinaron muy bueno y excelente respectivamente, lo que supone el $46.4 \%$ y el $29.1 \%$ del total de familiares (ver gráfica $5)$.

Gráfica 6. Preparación y formación del equipo de enfermería percibida por los familiares

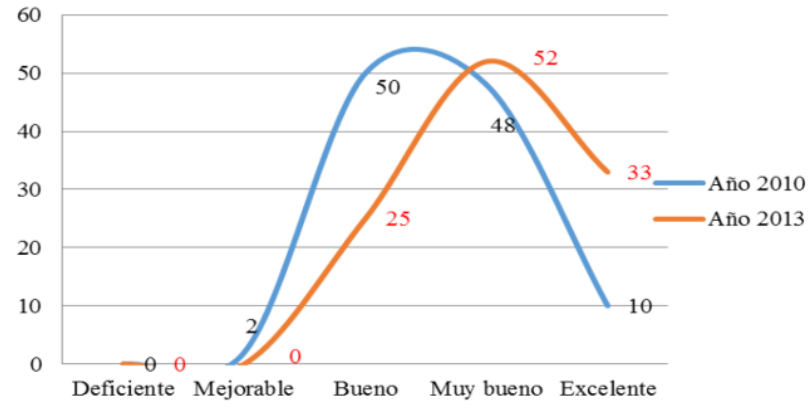

De los 110 familiares encuestados en 2010 ninguno percibió la formación y preparación del equipo de enfermería como deficiente, el 1.8\% de ellos opinaron mejorable, el $45.5 \%$ opinaron bueno, y el $43.6 \%$ y $9.1 \%$ opinaron muy bueno y excelente respectivamente.

En el año 2013 ningún familiar percibió la formación y preparación del equipo de enfermería como deficiente ni mejorable; 25 opinaron que bueno, lo que supone el $22.7 \%$. 52 y 33 familiares opinaron muy bueno y excelente respectivamente, lo que supone el $47.3 \%$ y el $30 \%$ del total de familiares (ver gráfica 6).

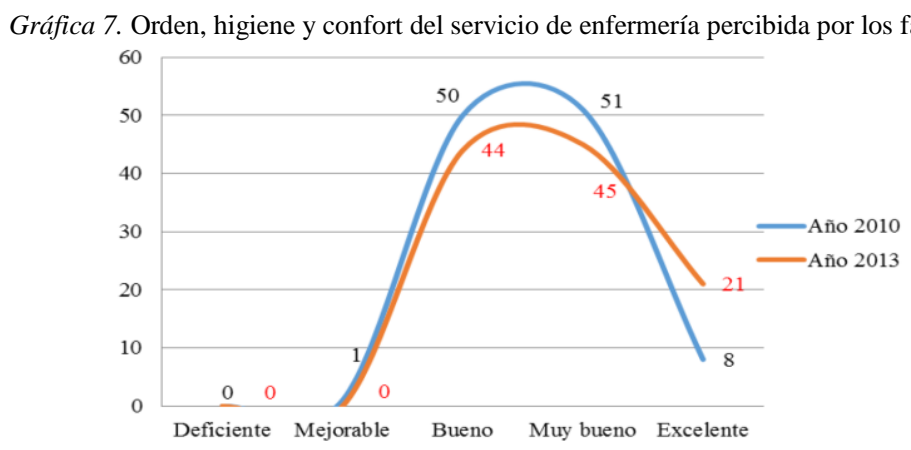

De los 110 familiares encuestados en 2010 ninguno percibió el orden, higiene y confort del servicio de enfermería como deficiente, el $0.9 \%$ de ellos opinaron mejorable, el $45.5 \%$ opinaron bueno, y el $46.3 \%$ y $7.2 \%$ opinaron muy bueno y excelente respectivamente. 
En el año 2013 ningún familiar percibió el orden, higiene y confort del servicio de enfermería como deficiente ni mejorable; 44 opinaron que bueno, lo que supone el $40 \% .45$ y 21 familiares opinaron muy bueno y excelente respectivamente, lo que supone el $40.1 \%$ y el $19.1 \%$ del total de familiares (ver gráfica 7 ).

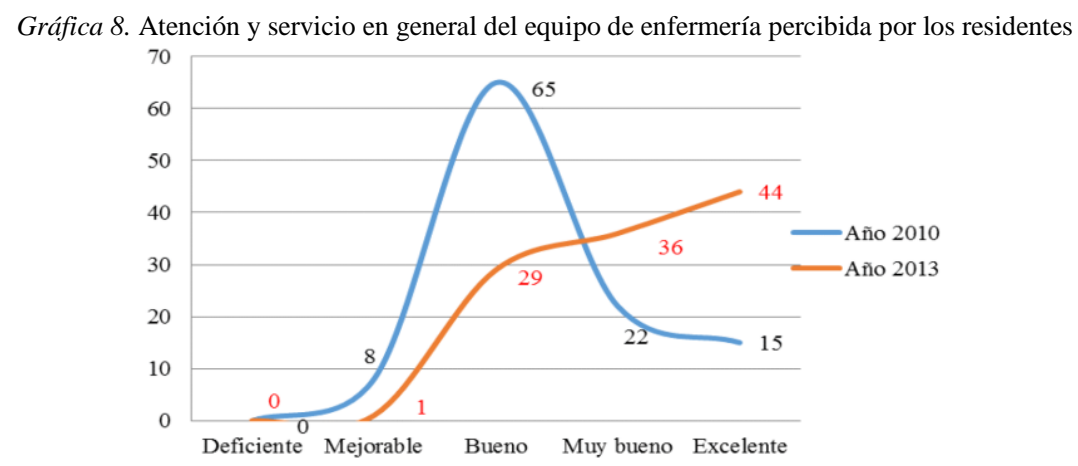

De los 110 familiares encuestados en 2010 ninguno percibió la atención y el servicio del equipo de enfermería como deficiente, el $7.3 \%$ de ellos opinaron mejorable, el $59.1 \%$ opinaron bueno, y el $20 \%$ y $13.7 \%$ opinaron muy bueno y excelente respectivamente.

En el año 2013 ningún familiar percibió la atención y servicio del equipo de enfermería como deficiente mientras que 1 opinó mejorable, lo que supone el $0.9 \% ; 29$ opinaron bueno, lo que supone el $26.4 \%$ y 36 y 44 familiares opinaron muy bueno y excelente respectivamente, lo que supone el $32.7 \%$ y el $40 \%$ del total de residentes (ver gráfica 8).

\section{DISCUSIÓN}

El presente estudio coincide con otros mencionados anteriormente en la importancia de implantar un Sistema de Gestión de Calidad como requisito fundamental para ofrecer cuidados de calidad, sirviendo como valiosa herramienta que siendo utilizada de forma interdisciplinar persiga la consecución de un equilibrio entre los recursos disponibles y la demanda de los usuarios, promoviendo un aumento en la satisfacción de estos mediante la mejora continua del servicio enfermero (Manzano, Vázquez, Gutiérrez y Rivero, 2011).

Ciertos ítems regulados por la norma del sistema de gestión como son la atención general del servicio de enfermería o la formación del personal se han visto claramente favorecidos por la implantación de la Norma UNE EN ISO 9001:2008 
produciéndose una evolución muy positiva tanto en la percepción de los residentes como en la de los familiares.

En cambio, otros ítems no regulados por dicha norma como son el orden, confort o higiene del servicio de enfermería apenas han variado a ojos de residentes y familiares, manteniéndose sin cambios las percepciones de estos a lo largo de los tres años.

En general el estudio muestra que la implantación de un sistema de gestión de calidad proporciona una herramienta útil al servicio de enfermería en residencias de personas mayores evidenciándose los efectos positivos que resultan de dicha implantación mediante encuestas de satisfacción e indicadores sanitarios (mejora la calidad en la práctica clínica, sirve como instrumento de participación a familiares y residentes, aporta información para mejorar,...).

Dicho esto concluimos que la calidad no debe ser considerada una tendencia actual y sin más relevancia, sino que por el contrario tiene que ser entendida como un modelo de vida que debería estar implantado en todas las organizaciones para dar una atención completa a nuestros mayores.

\section{REFERENCIAS}

Aenor. (2008). Norma UNE-EN ISO 9001:2008. Sistemas de gestión de la calidad. Requisitos. Madrid.

Cabarcas, N. (2011). Gestión de la calidad del cuidado de enfermería con principios éticos. Revista Ciencias Biomédicas, 2(1), 144-148.

Folstein, M.F., Folstein, S.E., McHugh, P.R y Fanjiang, G. (1975). "Mini-mental State” a practical method for grading the cognitive state of patients for the clinician. Journal of Psychiatric Researches, 12, 189-198.

Grupo de Trabajo sobre Gestión de Calidad de la Sociedad Española de Nefrología (SEN) (2006). Implantación de Sistemas de Gestión de Calidad en las unidades de Nefrología española. Nefrología, 26(2), 234-241.

Llecha J. (2008). La calidad en los servicios gerontológicos. Revista de Educación Social. (Consultado el 12-12-2013). Disponible en http://www.eduso.net

Manzano, B., Vázquez, C., Gutiérrez, E., Rivero, J. y Rivero, M. (2011). Gestión de la calidad en enfermería en un centro periférico de diálisis. Revista de la Sociedad Española de Enfermería Nefrológica, 14(1), 15-16.

Recibido: 28 de abril de 2014

Recepción Modificaciones: 26 de mayo de 2014

Aceptado: 18 de junio de 2014 